\title{
Solving Black-Schole Equation Using Standard Fractional Brownian Motion
}

\author{
Didier Alain Njamen Njomen ${ }^{1} \&$ Eric Djeutcha ${ }^{2}$ \\ ${ }^{1}$ Department of Mathematics and Computer's Science, Faculty of Science, University of Maroua, Cameroon \\ 2 Department of Mathematics, Higher Teachers' Training College, University of Maroua, Cameroon \\ Correspondence: Didier Alain Njamen Njomen, Department of Mathematics and Computer's Science, Faculty of Science, \\ University of Maroua, Po-Box 814, Cameroon.
}

Received: May 2, 2017 Accepted: June 5, 2017 Online Published: March 24, 2019

doi:10.5539/jmr.v11n2p142 URL: https://doi.org/10.5539/jmr.v11n2p142

\begin{abstract}
In this paper, we emphasize the Black-Scholes equation using standard fractional Brownian motion $B_{H}$ with the hurst index $H \in[0,1]$. N. Ciprian (Necula, C. (2002)) and Bright and Angela (Bright, O., Angela, I., \& Chukwunezu (2014)) get the same formula for the evaluation of a Call and Put of a fractional European with the different approaches. We propose a formula by adapting the non-fractional Black-Scholes model using a $\lambda_{H}$ factor to evaluate the european option. The price of the option at time $t \in] 0, T$ [ depends on $\lambda_{H}(T-t)$, and the cost of the action $S_{t}$, but not only from $t-T$ as in the classical model. At the end, we propose the formula giving the implied volatility of sensitivities of the option and indicators of the financial market.
\end{abstract}

Keywords: stock price, black-Scholes model, fractional Brownian motion, options, volatility

\section{Introduction}

Among the first systematic treatments of the option pricing problem has been the pioneering work of Black, Scholes and Merton (see Black, F. \& Myron, S. (1973)) who proposed the widely known, and extensively used, Black-Scholes (BS) model.

The BS model rests on the assumption that the natural logarithm of the stock price $\left(S_{t}\right)_{t>0}$ follows a random walk or diffusion with deterministic drift. However, this classical formula is quite often criticized.

In this paper, we was interested in modeling the long range dependence of asset returns using fractional Brownian motion.

The idea that stock returns could exhibit long range dependence was first suggested by Mandelbrot(see Mandelbrot, B. (1971)). Mandelbrot and Van Ness (Mandelbrot, B. \& Van Ness, J. (1968)) introduce the fractional Brownian motion (fbm), a non-semimartingale Gaussian process with long range dependence and dependent increments. Another important feature of this process is its dependence on a Hurst parameter, commonly used as a measure of market predictability. Market predictability may result from the dependence of the returns. Peters (Peters, E. E. (1996)) suggests that if a stock time series has a high Hurst exponent, then the stock will be less risky.

The application of fbm in finance was abandoned in its early years since this process is not a semimartingale which results in two problems: the first is defining a stochastic calculus with respect to fbm and the second is that arbitrage opportunities cannot be excluded in this model.

Actually there are several methods available which allow to construct a stochastic calculus for this process. The socalled Malliavin calculus(Nualart, D. (1995)) is based on a probabilistic and functional analytic notion of derivation and integration and allows a very efficient stochastic calculus for Gaussian processes which has already been successfully applied to mathematical models in finance (see Malliavin, P., \& Thalmaier, A. (2006)).

A second method, introduced in P. Vallois (Russo, F.M., \& Vallois, P.(1993)), defines three types of integrals (forward, backwards and symmetric) and gives a theory of stochastic integration through regularization. The advantage of regularization lies in the fact that this approach is natural and relatively simple, and easily connects to other approaches.

A third method is the so-called Wick product for constructing stochastic integrals, see F. Biagini (Biagini, F., Hu, Y., Øksendal, B., \& Zhang, T. (2008)). Arbitrage pricing theory is based on a fundamental result of Delbaen and Schachermayer (Delbaen, F., \& Schachermayer, W. (1994))known as the fundamental theorem of asset pricing which shows in full generality that no free lunch with vanishing risk" (NFLVR) is equivalent to the existence of an equivalent local martingale measure. A similar result has also been established by Ansel and Stricker (Ansel, J.P., \& Stricker, C. (1992)). 
In other words, there is no arbitrage if and only if there exists a martingale measure under which the discounted prices are martingales. Since fractional Brownian motion is not a semimartingale, a model in which the log prices are described by a fractional Brownian motion is not arbitrage-free, in the sense that there exists a strategy $\phi \in \mathcal{A}$ realizing a possibly non-zero gain by starting from a zero initial capital. L. Rogers (Rogers, L.C.G. (1997)) gives the mathematical argument for existence of arbitrage opportunities for this process.

Several attempts have been proposed in order to exclude arbitrage opportunities in the context of non-semimartingale models, like fractional Brownian motion. Cheridito (Cheridito, P. (2003)) suggests to forbid high frequency trading, and Guasoni, (Guasoni, P. (2006)) suggests to introduce transaction costs. However, none of these method were sufficient to resolve completely the problem. Even if arbitrage opportunities disappear, option pricing with these methods remains an open problem. In the other hand, Coviello and al. (Coviello, R., Cristina, D. G., \& Russo, F. (2011)) proposed to proceed by restricting the class of admissible strategies. In B. Mandelbrot and J. Van (Mandelbrot, B. \& Van Ness,J. (1968)) the authors proposed non-semimartingale models and also proceed by defining a class of admissible strategies for which the model is free of arbitrage. On the other hand, most of the recent literature dealing with modeling of financial assets assumes that the underlying dynamics of equity prices follows a diffusion process plus a jump process or Levy process in general. This is done to incorporate rare or extreme events not captured in Gaussian models.

A normalized fractional Brownian motion $B_{H}=\left\{B_{H}(t): 0 \in t<\infty\right\}$ with $0<H<1$ is uniquely characterized by the following properties:

1. if $B_{0}^{H}=0, \mathbb{P}-p s$,

2. it is a Gaussian process with $\mathbb{E}\left(B_{H}(t)\right)=0$ and $\mathbb{E}\left[\left(B_{t}^{H}\right)^{2}\right]=|t|^{2 H}, \forall t \in \mathbb{R}^{+}$,

3. the process $B^{H}$ has stationary increments,

4. the process $B^{H}$ has almost surely continuous path.

From the first three properties it follows that the covariance function is given by:

$$
\Gamma(s, t)=\frac{1}{2}\left[|s|^{2 H}+|t|^{2 H}-|s-t|^{2 H}\right]
$$

For Gaussian processes with $0<s<t$, the mean and covariance structure determine the finite-dimensional distributions uniquely.

In the literature there are many possible approaches to define such processes (see Bender, C., \& Marquardt, T. (2008); Lindstron, T., Øksendal, B., \& Uboe. (1992); Tikanmä ki, H., \& Mishura, Y.(2011)) showed for instance that this process has almost surely Hölder continuous paths of any order strictly less than $H-\frac{1}{2}$ for $H>\frac{1}{2}$, and with positive probability it has discontinuous sample paths for $H<\frac{1}{2}$. Hence, using this process to model jumps in asset returns may not be advantageous.

The paper is organized as follows: In section 2, we remind some results on fractional Itö integral and fractional Brownian properties, in section 3 we prove some results regarding the stochastic differential equation using fractional Brownian motion, in section 4 we apply these results in the study of the european options, in section 5 we apply these results in the study of the implied volatility european options and in section 6 we apply these results in the study of the sensibility indicator of the european options.

\section{Definition and Basic Properties}

In the pioneering work by Mandelbrot and N. Van (Mandelbrot, B. (1971)), fractional Brownian motion is defined by its stochastic representation

$$
B_{H}(t)=\frac{1}{\Gamma\left(H+\frac{1}{2}\right)}\left(\int_{-\infty}^{0}\left[(t-s)^{H-\frac{1}{2}}-(-s)^{H-\frac{1}{2}}\right] d B(s)+\int_{0}^{t}\left((t-s)^{H-\frac{1}{2}}\right) d B(s)\right)
$$

where $\Gamma$ represents the Gamma function $\Gamma(\alpha)=\int_{0}^{+\infty} x^{\alpha-1} \exp (-x) d x$ and $0<H<1$ is called the Hurst parameter (we soon see the connection with the Hurst parameter for self-similar processes). The integrator $B$ is a stochastic process, ordinary Brownian motion.

Note that $B$ is recovered by taking $H=\frac{1}{2}$ in (2). For later use, we assume that $B$ is defined on some probability space $(\Omega ; \mathcal{F} ; \mathbb{P})$. We remark that this representation in terms of an integral with respect to Brownian motion is non-unique (see Samorodnitsky, G. \& Taqqu, M.S. (1994)) for a different representation. 
Omitting details, the second moments of the stochastic integral in (2) can be computed by a standard formula, which leads to the fact that the variance of $B_{H}(t)$ is $V_{H} t^{2 H}$ for some constant $V_{H}$. From now on, we will always assume that we deal with normalized (or standardized) fractional Brownian motion, which has exactly variance $t^{2 H}$. We will use the same notation for the normalized process as before.

Therefore, we conclude from (1) that $\left\{B_{H}(a t): 0<t<1\right\}$ and $\left\{a^{H} B_{H}(t): 0<t<1\right\}$ have the same finite-dimensional distributions: fractional Brownian motion with Hurst parameter $H$ is self-similar with Hurst parameter $\mathrm{H}$.

In fact, fractional Brownian motion is the only Gaussian process with stationary increments that is self-similar(Cox, D.R. (1984)).

We define the incremental process $X=\left\{X_{k}: k=0 ; 1 ; \ldots\right\}$ of fractional Brownian motion, which is called fractional Gaussian noise, by $X_{k}=B_{H}(k+1)-B_{H}(k)$. It is clear that $X_{k}$ has a standard normal distribution for every $k$, but that there is (in general) no independence.

The corresponding autocovariance function $(\gamma()$.$) is of the form$

$$
\gamma(k)=\frac{1}{2}\left[|k-1|^{2 H}-2|k|^{2 H}+|k+1|^{2 H}\right],
$$

for $k \in \mathbb{Z}$. All the covariances are 0 (except, of course, for $k=0$ ). Since fractional Gaussian noise is a Gaussian process, this implies independence. This agrees with the properties of ordinary Brownian motion, which has independent increments.

By writing down the Taylor expansion at the origin of the function $h(x)=(1-x)^{2 H}-2+(1+x)^{2 H}$ and noting that $\gamma(k)=\frac{1}{2} k^{2 H} h\left(\frac{1}{k}\right)$ for $k \geq 1$, it is easily seen from (3) that

$$
\gamma(k) \sim H(2 H-1) k^{2 H-2}
$$

as $k \rightarrow \infty$. This implies long-range dependence for $\frac{1}{2}<H<1$ such that $\sum_{k=0}^{\infty} \gamma(k)=\infty$. It is important to know that the measurements on real traffic traces indeed suggest that $\frac{1}{2}<H<1$ (see Willinger, W., Taqqu, M.S., Leland, W.E., \& Wilson, D.V. (1995)).

Since we have noted that long-range dependence is closely related to self-similarity for $\frac{1}{2}<H<1$, it is natural to study whether fractional Gaussian noise is also a self-similar process.

The most important property associated with a fractional Brownian motion (Biagini, F., Yaozhong, H., Øksendal, B., \& Tusheng, Z.(2008a)), from Biagini and al. (Biagini, F., Yaozhong, H., Øksendal, B., \& Tusheng, Z.(2008b)) is summarized in the following proposition:

proposition 1 Let $B_{H}$ be a standard fractionnal Brownian motion

1. when $H=0.5$, the process coincides with the standard Brownian motion,

2. when $H \neq 0.5$, the process is not a semimartingale,

3. when $H>0.5$, the fractional Brownian motion is a long-range dependance and the short-range when $H<0.5$

lemma 1 (Fractionnal Itô's Formula) Let $H \in[0,1]$. Assume that $f(s, x): \mathbb{R} \times \mathbb{R} \rightarrow \mathbb{R}$ belongs to $C^{1,2}(\mathbb{R} \times \mathbb{R})$ and the random variable $f\left(t, B_{H}(t)\right), \int_{0}^{t} \frac{\partial f}{\partial s}\left(s, B_{H}(s)\right) d s$ and $\int_{0}^{t} \frac{\partial^{2} f}{\partial s^{2}}\left(s, B_{H}(s)\right) s^{2 H-1} d s$ all belong to $L^{2}(\mathbb{P})$ then

$$
f\left(t, B_{H}(t)\right)=f(0,0)+\int_{0}^{t} \frac{\partial f}{\partial s}\left(s, B_{H}(s)\right) d s+\int_{0}^{t} \frac{\partial f}{\partial x}\left(s, B_{H}(s)\right) d B_{H}(s)+H \int_{0}^{t} \frac{\partial^{2} f}{\partial x^{2}}\left(s, B_{H}(s)\right) s^{2 H-1} d s .
$$

Proof. For the proof of this lemma see, A. Inkaya (Inkaya, A. (2011)). In this paper, we consider a fractional Brownian motion and hurst parameter $H \in(0,1)$.

\section{Stochastic Differential Equation (SDE)}

Generally, we consider the Stochastics Differential Equation using fractional Brownian motion:

$$
d X_{t}=\sigma\left(t, X_{t}\right) d B_{H}(t)+\mu\left(t, X_{t}\right) d t
$$


where $\mu$ and $\sigma$ are constant.

The following result gives the solution of (6) in the general case

lemma 2 The coefficients $\sigma(t, x)=\sigma x$ and $\mu(t, x)=\mu x$ satisfy the overall conditions of lipschitz clearly and of restriction on growth, see A. Millet (Millet, A. (2000)). For all $t \geq 0$, the process fractionnal Brownian

$$
X(t)=X(0) \exp \left(\mu t+\sigma B_{H}(t)-\frac{1}{2} \sigma^{2} t^{2 H}\right)
$$

is a solution of (6).

Proof. Let us consider the fractional stochastic differential equation (6) which is the differential form of:

$$
X(t)=X(0)+\int_{0}^{t} \mu(s) X(s) d s+\int_{0}^{t} \sigma(s) X(s) d B_{H}(s),
$$

where $\mu(),. \sigma($.$) are locally bounded deterministic functions. By using the definition of fractional white noise in (S)^{*}$, we can write (6) as a differential equation in $(S)^{*}$ :

$$
\frac{d X(t)}{d t}=(\mu(t) X(t)+\sigma(t) X(t)) \diamond W_{H}(t)=X(t) \diamond\left[\mu(t)+\sigma(t) W_{H}(t)\right],
$$

When all the products are considered to be in the Wick's sense, (8) is the differential equation for the exponential.

The solution of this equation is obtained using the Wick calculus (Øksendal,B., Biagini,F., \&. al.(2008)):

$$
X(t)=X(0) \diamond \exp \diamond\left(\int_{0}^{t} \mu(s) d s+\int_{0}^{t} \sigma(s) d B_{H}(s)\right),
$$

where $\left.\int_{0}^{t} \beta(s) d B_{H}(s)\right)=\int_{\mathbb{R}} \beta(s) \mathbf{1}_{[0, t]}(s) d B_{H}(s) d B_{H}(s)$. Using the Wick exponential, our solution can be written as:

$$
X(t)=X(0) \diamond \exp \left(\int_{0}^{t} \sigma(s) d B_{H}(s)+\int_{0}^{t} \mu(s) d s-\frac{1}{2} \int_{\mathbb{R}}\left(M_{s}\left(\sigma(s) \mathbf{1}_{[0, t]}(s)\right)\right)^{2} d s\right),
$$

where $M_{s}$ is the operator $M$ acting on the variable $s$. If $X(0)=x$ is deterministic, the solution of the fractional stochastic differential equation (9) becomes

$$
X(t)=x \exp \left(\int_{0}^{t} \sigma(s) d B_{H}(s)+\int_{0}^{t} \mu(s) d s-\frac{1}{2} \int_{\mathbb{R}}\left(M_{s}\left(\sigma^{2}(s) \mathbf{1}_{[0, t]}(s)\right)\right)^{2} d s\right) .
$$

Furthermore if $\sigma(s)=\sigma, \mu(s)=\mu$ are constants, we obtain,

$$
X(t)=x \exp \left(\sigma B_{H}(t)+\mu t-\frac{1}{2} \sigma^{2} t^{2 H}\right) .
$$

\section{Presentation of the Black-Scholes Model}

\subsection{Factors Affecting the Price of Stock Options}

The following notations will be used in this paper :

- $S$ : The current stock price at time $t$;

- $V=V(t, S)$ : Value of option,

- $\sigma$ : The constant volatility of the stock price;

- $K$ : The strike price;

- $T$ : The time until expiration;

- $r$ : Risk-free interest rate. 


\subsection{Value of Stock Price at $t \in[0, T]$}

As far as our model is concerned, we consider a financial market comprising an asset known as constant rate risk $r$, such that $S_{0}^{0}=1$, so the stock price at $t$ is given by $S_{t}^{0}=e^{r t}$ where

$$
d S_{t}^{0}=r S_{t}^{0} d t,
$$

and an asset price of which $S_{t}$ at $t$ is a solution of the stochastic differential equation

$$
d S_{t}=\mu S_{t} d t+\sigma S_{t} d B_{H}(t)
$$

where $\left(B_{H}(t)\right)_{t \geq 0}$ is standard fractional Brownian motion, $S_{t}$ stock price at time $\mathrm{t}, \mu$ rate of return of the underlying stock price and $\sigma$ are constant volatility.

The solution of the equation (11) is given by the following result:

lemma 3 The fractionnal Brownian process $S_{t}$ define by:

$$
S_{t}=S_{0} \exp \left(\mu t+\sigma B_{H}(t)-\frac{1}{2} \sigma^{2} t^{2 H}\right)
$$

is solution of the SDE (11), $\mu$ is called the derive, and corresponds to a measure of the average growth rate of the price of the asset, and $\sigma$ is called volatility, and measures the fluctuation in the price of the financial asset considered.

Proof. We know that (11) admits a unique strong solution (see Millet, A. (2000)), since the coefficients $\sigma(t, x)=\sigma x$ and $\mu(t, x)=\mu x$ satisfy clearly the general conditions of lipschitz and of restriction on the growth.

For all $t \geq 0$, note

$$
X_{t}=S_{0} \exp \left(\mu t+\sigma B_{H}(t)-\frac{1}{2} \sigma^{2} t^{2 H}\right)
$$

We apply the fractional lemma 1 of Itô to the function

$$
f(t, x)=S_{0} \exp \left(\mu t+\sigma B_{H}(t)\right)-\frac{1}{2} \sigma^{2} t^{2 H},
$$

and the fractionnal Brownian $B_{H}$.

We deduce that

$$
X_{t}=S_{0}+\int_{0}^{t} \sigma X_{s} d B_{H}(s)+\int_{0}^{t}(\mu) X_{s} d s
$$

and by consequently $X$ is a solution of (11). The uniqueness of the strong solution entails that $X=S$, from where $S_{t}>0$, for all $t \geq 0$ p.s.

\subsection{Assumptions of the Black-Scholes Model}

The Black-Scholes model (Kavin, S. (2010); Arcadius, Y., \& Akossou, Y. J. (2014)) is based on a number of conditions which are based on the articles as follows:

$\left(C_{1}\right)$ the stock price underlying a fractional Brownian motion

$$
S(t)=S(0) \exp \left(\mu t+\sigma B_{H}(t)-\frac{1}{2} \sigma^{2} t^{2 H}\right)
$$

solution of $d S(t)=\mu S(t) d t+\sigma S(t) d B_{H}(t)$ where $\left\{B_{H}(t), t \geq 0\right\}$ is standard fractional Brownian motion;

$\left(C_{2}\right)$ no dividends are paid during the life of the option;

$\left(C_{3}\right)$ the steps are perfect and efficient;

$\left(C_{4}\right)$ no brokerage fee is levied.

$\left(C_{5}\right)$ interest rates remain constant and known;

$\left(C_{6}\right)$ the options are European-style 
$\left(C_{7}\right)$ the price fluctuations of the underlying are distributed according to a lognormal law.

\subsection{Fractionnal Black-Scholes Equation}

Given the conditions enumerated in the previous section, the fractional Black-Scholes equation allows to model the value of the option as a function of the time and the value of the action underlying. It is a partial differential equation (PDE) of the parabolic type two, and it is therefore necessary to specify an initial (or final) condition in time, and boundary conditions in space.

The following theorem enable to evaluate the value of the european option and constitue the first result of the fundamental paper:

Theorem 1 Under the conditions $\left(C_{1}\right)-\left(C_{7}\right)$, the Black-Scholes equation by fractionnal Brownian motion $(\mathrm{fbm})$ is defined by

$$
r S \frac{\partial V}{\partial S}(t, S)+\frac{\partial V}{\partial t}(t, S)+H t^{2 H-1} \sigma^{2} S^{2} \frac{\partial^{2} V}{\partial S^{2}}(t, S)-r V=0 .
$$

It is a partial differential equation (PDE) of two order, of the parabolic type, and it is necessary to specify an initial (or final) condition time, and boundary conditions in space.

Proof. We set the horizon $T>0$, and we want to give the price of one a financial asset that pays $h\left(S_{T}\right)$ on the date $T$. The case of a European call currency of $T$ and of the price of the year $K$ corresponds to case

$$
h(x)=(x-K)^{+} .
$$

We are a portfolio that includes $\alpha_{t}$ shares of the risk-free asset parts $S_{t}^{0}$, (the amount of wealth invested in this asset on the date $t$ is therefore $\alpha_{t} e^{r t}$ ), and $\beta_{t}$ shares of risky assets $S_{t}$. We assume that there is no transaction (purchase price or sale of assets), which can be sold short (that is to say that the coefficients $\alpha_{t}$ and $\beta_{t}$ can be negative), that the assets are indefinitely divisible, ( $\alpha_{t}$ and $\beta_{t}$ are real values) and that the trading is done continuously (from which money is not withdrawn before the date $\mathrm{T})$.

We want to find a portfolio that does not require of funds other than on date 0 , and which does not pay a dividend which does not withdraw money before the date $T$ ) whose value terminal is $h\left(S_{T}\right)$. The value of this portfolio at time $\mathrm{t}$ is given by:

$$
V_{t}=\alpha_{t} S_{t}^{0}+\beta_{t} S_{t}
$$

and in continuous time, equation (16) is defined by:

$$
d V_{t}=\alpha_{t} d S_{t}^{0}+\beta_{t} d S_{t} .
$$

by postponing (10) and (11) in (17) we obtain

$$
d V_{t}=\sigma \beta_{t} S_{t} d B_{H}(t)+\left[r V_{t}+\beta_{t} S_{t}(\mu-r)\right] d t,
$$

and we note that the initial value of the portfolio will be the value of the financial asset.

We assume that the $V_{t}$ value of the portfolio at the date $t$ is a deterministic function of time and value of the asset risk. Set $V_{t}=V\left(t, S_{t}\right)$. We apply the lemma 1 of Itô fractional by deriving $V_{t}$ such that

$$
\begin{aligned}
& d V_{t}=d V\left(t, S_{t}\right) . \\
& d V\left(t, S_{t}\right)= \frac{\partial V}{\partial x}\left(t, S_{t}\right) d S_{t}+\frac{\partial V}{\partial t}\left(t, S_{t}\right) d t+H \frac{\partial^{2} V}{\partial x^{2}}\left(t, S_{t}\right) t^{2 H-1}\left(d S_{t}\right)^{2} \\
&= \frac{\partial V}{\partial t}\left(t, S_{t}\right) d t+\frac{\partial V}{\partial x}\left(t, S_{t}\right)\left[\mu S_{t} d t+\sigma S_{t} d B_{H}(t)\right]+H \frac{\partial^{2} V}{\partial x^{2}}\left(t, S_{t}\right) t^{2 H-1}\left(\mu S_{t} d t+\sigma S_{t} d B_{H}(t)\right)^{2} \\
&=\frac{\partial V}{\partial t}\left(t, S_{t}\right) d t+\frac{\partial V}{\partial x}\left(t, S_{t}\right) \sigma S_{t} d B_{H}(t)+\frac{\partial V}{\partial x}\left(t, S_{t}\right) \mu S_{t} d t+H \frac{\partial^{2} V}{\partial x^{2}}\left(t, S_{t}\right) t^{2 H-1}\left(\mu^{2}\left(S_{t}\right)^{2}(d t)^{2}\right. \\
&\left.+\sigma^{2} S_{t}^{2}\left(d B_{H}(t)\right)^{2}+2 \sigma \mu S_{t}^{2} d t d B_{H}(t)\right)
\end{aligned}
$$


Yet

$$
(d t)^{2}=0, \quad d t d B_{H}(t)=0 \quad \text { et } \quad\left(d B_{H}(t)\right)^{2}=d t
$$

so we have

$$
d V_{t}=\left[\frac{\partial V}{\partial t}\left(t, S_{t}\right)+\mu S_{t} \frac{\partial V}{\partial x}\left(t, S_{t}\right)+H t^{2 H-1} \sigma^{2} S_{t}^{2} \frac{\partial^{2} V}{\partial x^{2}}\left(t, S_{t}\right)\right] d t+\sigma \frac{\partial V}{\partial x}\left(t, S_{t}\right) S_{t} d B_{H}(t)
$$

By using (18) and (19) and by identifying the martingale parts, we obtain:

$$
\beta_{t}=\frac{\partial V}{\partial x}\left(t, S_{t}\right)
$$

and identifying the martingale parts to finite variations, we obtain

$$
r V_{t}+\beta_{t} S_{t}(\mu-r)=\frac{\partial V}{\partial t}\left(t, S_{t}\right)+\mu S_{t} \frac{\partial V}{\partial x}\left(t, S_{t}\right)+H t^{2 H-1} \sigma^{2} S_{t}^{2} \frac{\partial^{2} V}{\partial x^{2}}\left(t, S_{t}\right),
$$

and simplifying (21), we obtain:

$$
r S_{t} \frac{\partial V}{\partial x}\left(t, S_{t}\right)+\frac{\partial V}{\partial t}\left(t, S_{t}\right)+H t^{2 H-1} \sigma^{2} S_{t}^{2} \frac{\partial^{2} V}{\partial x^{2}}\left(t, S_{t}\right)-r V_{t}=0,
$$

hence the proof of the theorem.

The equation (15) obtained from the theorem 1 is the same equation obtained by Necula (Necula, C. (2002)) and Bright and Angela (Bright, O., Angela, I., \& Chukwunezu (2014)). These three authors take their inspiration from the theory of wick product and use fourier transform.

\subsection{Analytic Solution of the Fractional Black-Scholes Equation}

The theoretical price of a call option, which gives the right but not the obligation to purchase the asset $S$ to the value $K$ on the date $T$, is characterized by its pay-off :

$$
(S-E)^{+}=\max (S-K ; 0)
$$

it is given by hope risk-neutral probability of the updated pay-off terminal :

$$
C(S, t)=\mathbb{E}\left(\text { payoff } \times e^{-r T}\right)
$$

similarly, the theoretical price of a put option is of pay off :

$$
(K-S)^{+}=\max (K-S ; 0)
$$

and is given by the expectation under probability Payoff terminal updated :

$$
P(S, t)=\mathbb{E}\left(\text { payoff } \times e^{-r T}\right)
$$

The analytic resolution of the (15) equation gives the theorical price of an option. Thus, for a call option, we obtain the following theorem that enable to calculate the value of an european call option and constitue the second result of fundamental paper:

Theorem 2 The value of the european call at time $t \in] 0, T$ [is given by:

$$
C(S, t)=S F\left(d_{1}^{\lambda_{H}}\right)-K e^{-r(T-t)} F\left(d_{2}^{\lambda_{H}}\right),
$$

that is the solution of equation (15) with $F($.$) the distribution function of a Gaussian law \mathcal{N}(0.1)$ defined by

$$
F(d)=\frac{1}{\sqrt{2 \pi}} \int_{-\infty}^{d} e^{-\frac{s 2}{2}} d s
$$

$d_{1}^{\lambda_{H}}$ and $d_{2}^{\lambda_{H}}$ are respectively define by

$$
\begin{gathered}
d_{1}^{\lambda_{H}}=\frac{\ln \left(\frac{S}{K}\right)+\left(r+\frac{\lambda_{H}}{2} \sigma^{2}\right)(T-t)}{\sigma \sqrt{\lambda_{H}(T-t)}}, \\
d_{2}^{\lambda_{H}}=\frac{\ln \left(\frac{S}{K}\right)+\left(r-\frac{\lambda_{H}}{2} \sigma^{2}\right)(T-t)}{\sigma \sqrt{\lambda_{H}(T-t)}} \text { where } \lambda_{H}=2 H t^{2 H-1} .
\end{gathered}
$$


Proof. we establish the relation between the fraction equation Black-Scholes equation and the heat equation we consider the case of an option purchase (call). The equation (15) is therefore written in the form:

$$
r S \frac{\partial C}{\partial S}(t, S)+\frac{\partial C}{\partial t}(t, S)+H t^{2 H-1} \sigma^{2} S^{2} \frac{\partial^{2} C}{\partial S^{2}}(t, S)-r C=0
$$

with boundary conditions :

$$
C(0, t)=0, \forall t \text { et } C(S, t) \sim S, S \rightarrow+\infty,
$$

and the final condition:

$$
C(S, T)=\max (S-K, 0), \quad \forall S .
$$

And proceeding to changes of the following variables:

$$
S=K e^{x} ; t=T-\frac{2 \tau}{\sigma^{2}}
$$

and

$$
C(S, \tau)=K v(x, \tau),
$$

The different derivatives of (27) allow us to define by the following system:

$$
\left\{\begin{array}{l}
S \frac{\partial C}{\partial S}=K \frac{\partial v}{\partial x} \\
\frac{\partial C}{\partial t}=-\frac{1}{2} K \sigma^{2} \frac{\partial v}{\partial \tau} \\
S^{2} \frac{\partial^{2} C}{\partial S^{2}}=K\left(\frac{\partial^{2} v}{\partial x^{2}}-\frac{\partial v}{\partial x}\right) \\
C(S, \tau)=K v(x, \tau) .
\end{array}\right.
$$

By providing each of the expressions of (29) in the equation (27) of black-scholes, we obtain the following equation:

$$
-\frac{K \sigma^{2}}{2} \frac{\partial v}{\partial \tau}+r K \frac{\partial v}{\partial x}+H t^{2 H-1} \sigma^{2}\left(K \frac{\partial^{2} v}{\partial x^{2}}-K \frac{\partial v}{\partial x}\right)-r K v=0
$$

by dividing (30) by $\frac{K}{2} \sigma^{2}$, the equation (30) becomes

$$
-\frac{\partial v}{\partial \tau}+\frac{2 r}{\sigma^{2}} \frac{\partial v}{\partial x}+2 H t^{2 H-1}\left(\frac{\partial^{2} v}{\partial x^{2}}-\frac{\partial v}{\partial x}\right)-\frac{2 r}{\sigma^{2}} v=0
$$

By setting $\xi=\frac{2 r}{\sigma^{2}}$, the equation (31) becomes:

$$
\frac{\partial v}{\partial \tau}-2 H t^{2 H-1} \frac{\partial^{2} v}{\partial x^{2}}+\left(2 H t^{2 H-1}-\xi\right) \frac{\partial v}{\partial x}+\xi v=0
$$

Thus, with these changes of variables, we thus pass from the domain $t \in[0, T]$ and $S \in\left[0,+\infty\left[\right.\right.$ to domain $\tau \in\left[0, \frac{1}{2} \sigma^{2} T\right]$ and $S \in \mathbb{R}$. The conditions to the limits $C(T, S)=\max (S-E, 0)$ becomes:

$$
K v(t=0, x)=\max \left(K e^{x}-K, 0\right) \Leftrightarrow v(t=0, x)=\max \left(e^{x}-1,0\right) .
$$

By setting

the equation (32) becomes:

$$
\lambda_{H}=2 H\left(T-\frac{2 \tau}{\sigma^{2}}\right)^{2 H-1}
$$

$$
\frac{\partial v}{\partial \tau}=\lambda_{H} \frac{\partial^{2} v}{\partial x^{2}}+\left(-\xi+\lambda_{H}\right) \frac{\partial v}{\partial x}+\xi v=0 .
$$

We proceed to a second change of variables to obtain a generalized heat equation by setting again

$$
v(x, \tau)=e^{\alpha x+\beta \tau} u(x, \tau),
$$

We obtain the following new system:

$$
\left\{\begin{array}{l}
\frac{\partial v}{\partial \tau}=\beta e^{\alpha x+\beta \tau} u+e^{\alpha x+\beta \tau} \frac{\partial u}{\partial \tau} \\
\frac{\partial v}{\partial x}=\alpha e^{\alpha x+\beta \tau} u+e^{\alpha x+\beta \tau} \frac{\partial u}{\partial x} \\
\frac{\partial^{2} v}{\partial x^{2}}=\alpha^{2} e^{\alpha x+\beta \tau} u+2 \alpha e^{\alpha x+\beta \tau} \frac{\partial u}{\partial x}+e^{\alpha x+\beta \tau} \frac{\partial^{2} u}{\partial x^{2}}
\end{array}\right.
$$


By plotting the expressions of(36) into (34) and simplifying by $e^{\alpha x+\beta \tau}$, we obtain

$$
\frac{\partial u}{\partial \tau}=\lambda_{H} \frac{\partial^{2} u}{\partial x^{2}}+\left[2 \lambda_{H} \alpha+\xi-\lambda_{H}\right] \frac{\partial u}{\partial x}+\left(\lambda_{H} \alpha^{2}+\left[\xi-\lambda_{H}\right) \alpha-\xi-\beta\right] u .
$$

To be reduced to the case of the heat equation, we must eliminate in (37) the terms in $u$ and $\frac{\partial u}{\partial x}$ we solve the following system:

$$
\left\{\begin{array}{r}
\lambda_{H} \alpha^{2}+\left(\xi-\lambda_{H}\right) \alpha-\xi-\beta=0 \\
2 \lambda_{H} \alpha+\xi-\lambda_{H}=0
\end{array}\right.
$$

so, we have

$$
\left\{\begin{array}{l}
\alpha=-\frac{\xi-\lambda_{H}}{2 \lambda_{H}} \\
\beta=-\frac{\left(\xi+\lambda_{H}\right)^{2}}{4 \lambda_{H}} .
\end{array}\right.
$$

By plotting the $\alpha$ and $\beta$ coefficients of (39) into (35) we are getting

$$
u(x, \tau)=e^{\frac{\xi-\lambda_{H}}{2 \lambda_{H}} x-\frac{\left(\xi+\lambda_{H}\right)^{2}}{4 \lambda_{H}} \tau} v(x, \tau) .
$$

Thus, for these particular values of $\alpha$ and $\beta$, the equation (37) that we obtain is known as the heat equation and is defined by:

the exact solution of (41) is then

$$
\frac{\partial u}{\partial \tau}=\lambda_{H} \frac{\partial^{2} u}{\partial x^{2}}
$$

$$
u(x, \tau)=\frac{1}{2 \sqrt{\pi \lambda_{H} \tau}} \int_{-\infty}^{+\infty} u_{0}(s) e^{-\frac{(s-x)^{2}}{4 \lambda^{\tau} \tau}} d s,
$$

and which has as its initial condition

$$
u(x, 0)=u_{0}(x)=\max \left(e^{\frac{\xi+\lambda_{H}}{2 \lambda_{H}} x}-e^{\frac{\xi-\lambda_{H}}{2 \lambda_{H}} x}, 0\right) .
$$

By putting (42) in (40) we obtain

$$
v(x, \tau)=u(x, \tau) \times e^{-\frac{\xi+\lambda_{H}}{2 \lambda_{H}} x+\frac{\left(\xi+\lambda_{H}\right)^{2}}{4 \lambda_{H}} \tau}
$$

and (44) in (28) give us

$$
C(S, \tau)=K v(x, \tau) .
$$

That is the end of this proof.

The analytic resolution of the (15) equation gives the theory price of an option. Thus, for a put option, we obtain the following theorem that enable to calculate the value of the put european option and constitute the third result of a fundamental paper:

Theorem 3 The value of the european put at time $t \in] 0, T$ [ is given by:

$$
P(S, t)=-S F\left(-d_{1}^{\lambda_{H}}\right)+K e^{-r(T-t)} F\left(-d_{2}^{\lambda_{H}}\right),
$$

that is the solution of equation (15) with $F($.) the distribution function of a Gaussian law $\mathcal{N}(0.1)$ defined by

$$
F(d)=\frac{1}{\sqrt{2 \pi}} \int_{-\infty}^{d} e^{-\frac{s 2}{2}} d s
$$

$d_{1}^{\lambda_{H}}$ and $d_{2}^{\lambda_{H}}$ are respectively defining by

where $\lambda_{H}=2 H t^{2 H-1}$.

$$
\begin{aligned}
& d_{1}^{\lambda_{H}}=\frac{\ln \left(\frac{S}{K}\right)+\left(r+\frac{\lambda_{H}}{2} \sigma^{2}\right)(T-t)}{\sigma \sqrt{\lambda_{H}(T-t)}}, \\
& d_{2}^{\lambda_{H}}=\frac{\ln \left(\frac{S}{K}\right)+\left(r-\frac{\lambda_{H}}{2} \sigma^{2}\right)(T-t)}{\sigma \sqrt{\lambda_{H}(T-t)}}
\end{aligned}
$$


Proof. The proof is similar to that of the previous theorem.

Remark 1 We notice that the price of purchase of an european option at given moment $t \in] 0, T\left[\right.$ depends on $\lambda_{H}^{H}(T-t)$, that can be justified by the fact the fbm has a long-range dependance and the cost of option $S(t)$.

\subsection{Conditions to the Limits}

\subsubsection{Case of Call}

In the case of a call at maturity (see Kavin, S. (2010); Arcadius, Y., \& Akossou, Y. J. (2014)) i.e. when $t=T$, the value of the call is given by Formula:

$$
C(S, T)=\max (S-K, 0), \forall S .
$$

If $S$ is less than the exercise price at the end of the option, it is to avoid losing $K-S$. Otherwise, the benefit of the call is $S-K$. Therefore the field of study in space (variable $S$ ) is theoretically $[0,+\infty[$, and therefore the boundary conditions the function $C$. If $S=0$, then the forward profit is necessarily no. There is therefore no interest in exercising the call option in this cases, even if there is still time before it expires. So we have the Condition:

$$
C(0, t)=0, \forall t
$$

If, on the other hand, the price of the share increases considerably $S \rightarrow+\infty$, it is obvious that the option will be exercised and the exercise price of the option will be negligible. We have the following condition:

$$
C(S, t) \sim S, S \rightarrow+\infty
$$

\subsubsection{Case of Put}

In the case of a put at maturity (see Kavin, S. (2010); Arcadius, Y., \& Akossou, Y. J. (2014)),

$$
P(S, T)=\max (K-S ; 0), \forall t .
$$

If $S$ is above the exercise price at the end of the option, it is to exercise the option (under penalty of losing $S-K$ ). Otherwise, the benefit of the call is $K-S$. The domain Of study in space (variable $S$ ) is always theoretically $[0,+\infty$ [. It is therefore necessary to set the boundary conditions on Function $P$. If the price of the share increases considerably $(S \rightarrow+\infty)$, it is obvious that the option will not be exercised. We have the following condition:

$$
P(S, t) \sim 0, S \rightarrow+\infty
$$

If on the contrary $S=0$, then the benefit to term is necessarily $K$.

In the following sections 5 and 6 we set $d_{1}=d_{1}^{\lambda_{H}}$ and $d_{2}=d_{2}^{\lambda_{H}}$ of Theorem 2 to simplify our results.

\section{Estimation of Volatility}

Volatility is essential in financial mathematics for calculate the price of an option.

It measures the volatility of the price of a financial asset. The greater the volatility, the more volatile the asset is, if the volatility is zero, the value can be accurately of the assets in the future. There are two methods of calculating the volatility:

- implicit volatility;

- historical volatility.

In this paper, we only express the implicit volatility, using observed the price of options $C_{t}$ and reversing the BlackScholes formula, we can find the parameter $\Sigma$ that we identify to $\sigma_{\lambda_{H}}$. and the following corollary gives the formula for calculating the implicit volatility. This corollary is the fourth fondamental result of this paper:

Corollary 1 The implicit volatility of the option price at $t \in] 0, T[$ is defined by:

$$
\sigma_{\lambda_{H}}=\sqrt{\frac{\ln \left(\frac{K}{S}\right)-r(T-t)}{\lambda_{H}(T-t)\left(E_{\chi}-\frac{1}{2}\right)}} \text { with } \lambda_{H}=2 H t^{2 H-1} .
$$


Proof. Indeed, let's take the Black-Scholes formula for a call-european, so we have:

$$
\begin{aligned}
S F^{\prime}\left(d_{1}\right) & =\frac{S}{\sqrt{2 \pi}} e^{-\frac{1}{2}\left[\frac{\ln \left(\frac{S}{R}\right)+\left(r+\frac{\lambda_{H}}{2} \sigma^{2}\right)(T-t)}{\sigma \sqrt{\lambda_{H}(T-t)}}\right]^{2}} \\
& =\frac{S}{\sqrt{2 \pi}} e^{-\frac{1}{2}\left[\frac{\left(\ln \left(\frac{S}{K}\right)\right)^{2}+\left(r+\frac{\lambda_{H}}{2} \sigma^{2}\right)^{2}(T-t)^{2}+2 \ln \left(\frac{S}{K}\right)\left(r+\frac{\lambda_{H}}{2} \sigma^{2}\right)(T-t)}{\sigma^{2} \lambda_{H}(T-t)}\right]} \\
& =\frac{K}{\sqrt{2 \pi}} e^{\ln \left(\frac{S}{K}\right)} e^{-\frac{1}{2}\left[\frac{\left(\ln \left(\frac{S}{K}\right)\right)^{2}+\left(r+\frac{\lambda_{H}}{2} \sigma^{2}\right)^{2}(T-t)^{2}+2 \ln \left(\frac{S}{K}\right)\left(r+\frac{\lambda_{H}}{2} \sigma^{2}\right)(T-t)}{\sigma^{2} \lambda_{H}(T-t)}\right]} \\
& =\frac{K}{\sqrt{2 \pi}} e^{\ln \left(\frac{S}{K}\right)-\frac{1}{2}\left[\frac{\left(\ln \left(\frac{S}{K}\right)\right)^{2}+\left(r+\frac{\lambda_{H}}{2} \sigma^{2}\right)^{2}(T-t)^{2}+2 \ln \left(\frac{S}{K}\right)\left(r+\frac{\lambda_{H}}{2} \sigma^{2}\right)(T-t)}{\sigma^{2} \lambda_{H}(T-t)}\right]} \\
& =\frac{K}{\sqrt{2 \pi}} e^{\frac{K \sigma^{2} \lambda_{H}(T-t) \ln \left(\frac{S}{K}\right)-\left(\ln \left(\frac{S}{K}\right)\right)^{2}-\left(r+\frac{\lambda_{H}}{2} \sigma^{2}\right)^{2}(T-t)^{2}-2 \ln \left(\frac{S}{K}\right)\left(r+\frac{\lambda_{H}}{2} \sigma^{2}\right)(T-t)}{2 \sigma^{2} \lambda_{H}(T-t)}} \\
& =\frac{K}{\sqrt{2 \pi}} e^{-\frac{-\sigma^{2} \lambda_{H}(T-t) \ln \left(\frac{S}{R}\right)+2 \ln \left(\frac{S}{R}\right) r(T-t)+\left(\ln \left(\frac{S}{K}\right)\right)^{2}+\left(r+\frac{\lambda_{H}}{2} \sigma^{2}\right)^{2}(T-t)^{2}}{2 \sigma^{2} \lambda_{H}(T-t)}} \\
& =\frac{K}{\sqrt{2 \pi}} e^{-\frac{\left(\ln \left(\frac{S}{K}\right)\right)^{2}+\left(r+\frac{\lambda_{H}}{2} \sigma^{2}\right)^{2}(T-t)^{2}+2 \ln \left(\frac{S}{K}\right)\left(r-\frac{\sigma^{2} \lambda_{H}}{2}\right](T-t)}{2 \sigma^{2} \lambda_{H}(T-t)}} .
\end{aligned}
$$

and so $S F^{\prime}\left(d_{1}\right)=K e^{-r(T-t)} F^{\prime}\left(d_{2}\right)$ and $d_{1}=d_{2}+\sigma \sqrt{\lambda_{H}(T-t)}$

$$
\begin{gathered}
C^{\prime}=\frac{\partial C}{\partial \ln (S)}=S F\left(d_{1}\right)+\frac{S F^{\prime}\left(d_{1}\right)}{\sigma \sqrt{\lambda_{H}(T-t)}}-K e^{-r(T-t)} \frac{F^{\prime}\left(d_{2}\right)}{\sigma \sqrt{\lambda_{H}(T-t)}} \\
C^{\prime \prime}=\frac{\partial^{2} C}{\partial \ln ^{2}(S)}=S F\left(d_{1}\right)+\frac{2 S F^{\prime}\left(d_{1}\right)}{\sigma \sqrt{\lambda_{H}(T-t)}}+\frac{S F^{\prime \prime}\left(d_{1}\right)}{\left(\sigma \sqrt{\lambda_{H}(T-t)}\right)^{2}}-\frac{K e^{-r(T-t)} F^{\prime \prime}\left(d_{2}\right)}{\left(\sigma \sqrt{\lambda_{H}(T-t)}\right)^{2}}
\end{gathered}
$$

By setting

$$
\chi=C^{\prime \prime}-C^{\prime}
$$

we obtain:

$$
\begin{aligned}
\chi & =\frac{S F^{\prime}\left(d_{1}\right)}{\sigma \sqrt{\lambda_{H}(T-t)}}+\frac{S F^{\prime \prime}\left(d_{1}\right)}{\left(\sigma \sqrt{\lambda_{H}(T-t)}\right)^{2}}-\frac{K e^{-r(T-t)} F^{\prime \prime}\left(d_{2}\right)}{\left(\sigma \sqrt{\lambda_{H}(T-t)}\right)^{2}}+\frac{K e^{-r(T-t)} F^{\prime}\left(d_{2}\right)}{\sigma \sqrt{\lambda_{H}(T-t)}} \\
& =\frac{S F^{\prime}\left(d_{1}\right)}{\sigma \sqrt{\lambda_{H}(T-t)}}+\frac{S F^{\prime \prime}\left(d_{1}\right)}{\left(\sigma \sqrt{\lambda_{H}(T-t)}\right)^{2}}-\frac{K e^{-r(T-t)} F^{\prime \prime}\left(d_{2}\right)}{\left(\sigma \sqrt{\lambda_{H}(T-t)}\right)^{2}}+\frac{S F^{\prime}\left(d_{1}\right)}{\sigma \sqrt{\lambda_{H}(T-t)}} \\
& =\frac{2 S F^{\prime}\left(d_{1}\right)}{\sigma \sqrt{\lambda_{H}(T-t)}}+\frac{S F^{\prime \prime}\left(d_{1}\right)}{\left(\sigma \sqrt{\lambda_{H}(T-t)}\right)^{2}}-\frac{K e^{-r(T-t)} F^{\prime \prime}\left(d_{2}\right)}{\left(\sigma \sqrt{\lambda_{H}(T-t)}\right)^{2}} \\
& =\frac{2 S F^{\prime}\left(d_{1}\right)}{\sigma \sqrt{\lambda_{H}(T-t)}}-\frac{d_{1} S F^{\prime}\left(d_{1}\right)}{\left(\sigma \sqrt{\lambda_{H}(T-t)}\right)^{2}}+\frac{K d_{2} e^{-r(T-t)} F^{\prime}\left(d_{2}\right)}{\left(\sigma \sqrt{\lambda_{H}(T-t)}\right)^{2}} \\
& =\frac{2 S F^{\prime}\left(d_{1}\right)}{\sigma \sqrt{\lambda_{H}(T-t)}}+\frac{K d_{2} e^{-r(T-t)} F^{\prime}\left(d_{2}\right)-d_{1} S F^{\prime}\left(d_{1}\right)}{\left(\sigma \sqrt{\lambda_{H}(T-t)}\right)^{2}}
\end{aligned}
$$

Yet

$$
d_{1}=d_{2}+\sigma \sqrt{\lambda_{H}(T-t)}
$$


So

$$
\begin{aligned}
\chi & =\frac{2 S F^{\prime}\left(d_{1}\right)}{\sigma \sqrt{\lambda_{H}(T-t)}}+\frac{K d_{2} e^{-r(T-t)} F^{\prime}\left(d_{2}\right)-d_{1} S F^{\prime}\left(d_{1}\right)}{\left(\sigma \sqrt{\lambda_{H}(T-t)}\right)^{2}} \\
& =\frac{2 S F^{\prime}\left(d_{1}\right)}{\sigma \sqrt{\lambda_{H}(T-t)}}+\frac{K d_{2} e^{-r(T-t)} F^{\prime}\left(d_{2}\right)-d_{1} K d_{2} e^{-r(T-t)} F^{\prime}\left(d_{2}\right)}{\left(\sigma \sqrt{\lambda_{H}(T-t)}\right)^{2}} \\
& =\frac{2 S F^{\prime}\left(d_{1}\right)}{\sigma \sqrt{\lambda_{H}(T-t)}} \\
& +\frac{K d_{2} e^{-r(T-t)} F^{\prime}\left(d_{2}\right)}{\left(\sigma \sqrt{\lambda_{H}(T-t)}\right)^{2}} \\
& -\frac{\left(d_{2}+\sigma \sqrt{\lambda_{H}(T-t)}\right) K d_{2} e^{-r(T-t)} F^{\prime}\left(d_{2}\right)}{\left(\sigma \sqrt{\lambda_{H}(T-t)}\right)^{2}} \\
& =\frac{2 S F^{\prime}\left(d_{1}\right)}{\sigma \sqrt{\lambda_{H}(T-t)}}-\frac{\left.K \sigma \sqrt{\lambda_{H}(T-t)}\right) e^{-r(T-t)} F^{\prime}\left(d_{2}\right)}{\left(\sigma \sqrt{\lambda_{H}(T-t)}\right)^{2}} \\
& =\frac{2 S F^{\prime}\left(d_{1}\right)}{\sigma \sqrt{\lambda_{H}(T-t)}}-\frac{K e^{-r(T-t)} F^{\prime}\left(d_{2}\right)}{\sigma \sqrt{\lambda_{H}(T-t)}} \\
& =\frac{2 S F^{\prime}\left(d_{1}\right)}{\sigma \sqrt{\lambda_{H}(T-t)}}-\frac{S F^{\prime}\left(d_{1}\right)}{\sigma \sqrt{\lambda_{H}(T-t)}} \\
& =\frac{S F^{\prime}\left(d_{1}\right)}{\sigma \sqrt{\lambda_{H}(T-t)}} \cdot \\
&
\end{aligned}
$$

we have

$$
\chi=\frac{S F^{\prime}\left(d_{1}\right)}{\sigma \sqrt{\lambda_{H}(T-t)}} .
$$

Let the elasticity of the auxiliary function $\chi$ be compared with $S$

$$
E_{\chi}=\frac{\partial \ln |\chi|}{\partial \ln (S)} .
$$

Hence

$$
\begin{aligned}
\ln |\chi| & =\ln \left(S F^{\prime}\left(d_{1}\right)\right)-\ln \left(\sigma \sqrt{\lambda_{H}(T-t)}\right) \\
& =\ln \left(\frac{S}{\sqrt{2 \pi}} e^{-\frac{d_{1}^{2}}{2}}\right)-\ln \left(\sigma \sqrt{\lambda_{H}(T-t)}\right) \\
& =\ln (S)-\ln (\sqrt{2 \pi})-\frac{d_{1}^{2}}{2}-\ln \left(\sigma \sqrt{\lambda_{H}(T-t)}\right) .
\end{aligned}
$$


so

$$
\begin{aligned}
E_{\chi} & =1-\frac{1}{2} \frac{\partial\left(d_{1}^{2}\right)}{\partial \ln (S)} \\
& =1-\frac{d_{1}}{\sigma \sqrt{\lambda_{H}(T-t)}} \\
& =\frac{1}{2}+\frac{1}{2}+\frac{r}{\lambda_{H} \sigma^{2}}-\frac{r}{\lambda_{H} \sigma^{2}}-\frac{d_{1}}{\sigma \sqrt{\lambda_{H}(T-t)}} \\
& =\frac{1}{2}+\frac{1}{2}-\left[\frac{\ln \left(\frac{S}{K}\right)+\left(r+\frac{\lambda_{H} \sigma^{2}}{2}\right)(T-t)}{\sigma^{2} \lambda_{H}(T-t)}\right]-\frac{r}{\lambda_{H} \sigma^{2}}+\frac{r}{\lambda_{H} \sigma^{2}} \\
& =\frac{1}{2}+\left[\frac{1}{2}+\frac{r}{\lambda_{H} \sigma^{2}}-\frac{\ln \left(\frac{S}{K}\right)}{\sigma^{2} \lambda_{H}(T-t)}-\frac{\left(r+\frac{\lambda_{H} \sigma^{2}}{2}\right)}{\lambda_{H} \sigma^{2}}\right]-\frac{r}{\lambda_{H} \sigma^{2}} \\
& =\frac{1}{2}-\frac{r}{\lambda_{H} \sigma^{2}}+\left[\frac{1}{2}+\frac{r}{\lambda_{H} \sigma^{2}}-\frac{\ln \left(\frac{S}{K}\right)}{\sigma^{2} \lambda_{H}(T-t)}-\frac{r}{\lambda_{H} \sigma^{2}}-\frac{1}{2}\right] \\
& =\frac{1}{2}-\frac{r}{\lambda_{H} \sigma^{2}}-\frac{\ln \left(\frac{S}{K}\right)}{\sigma^{2} \lambda_{H}(T-t)} \\
& =\frac{1}{2}-\frac{r}{\lambda_{H} \sigma^{2}}+\frac{\ln \left(\frac{K}{S}\right)}{\sigma^{2} \lambda_{H}(T-t)},
\end{aligned}
$$

Therefore

$$
\begin{aligned}
E_{\chi} & =\frac{1}{2}-\frac{r}{\lambda_{H} \sigma^{2}}+\frac{\ln \left(\frac{K}{S}\right)}{\sigma^{2} \lambda_{H}(T-t)} \\
& =\frac{1}{2}+\frac{1}{\sigma^{2}}\left[\frac{\ln \left(\frac{K}{S}\right)-r(T-t)}{\lambda_{H}(T-t)}\right]
\end{aligned}
$$

let

$$
E_{\chi}-\frac{1}{2}=\frac{1}{\sigma^{2}}\left[\frac{\ln \left(\frac{K}{S}\right)-r(T-t)}{\lambda_{H}(T-t)}\right]
$$

that is

$$
\sigma^{2}=\frac{\ln \left(\frac{K}{S}\right)-r(T-t)}{\lambda_{H}(T-t)\left(E_{\chi}-\frac{1}{2}\right)} .
$$

From where

$$
\sigma=\sqrt{\frac{\ln \left(\frac{K}{S}\right)-r(T-t)}{\lambda_{H}(T-t)\left(E_{\chi}-\frac{1}{2}\right)}} .
$$

so $\sigma_{\lambda_{H}}=\sigma$. That is the end of this proof

\section{The Sensitivities of the Option}

In this paper, we define the 5 sensitivities estimators of the option by:

- $\operatorname{Delta}(\Delta)$ :calls and puts represents the derivative of the price of an option over the underlying,

- $\operatorname{Gamma}(\Gamma)$ : represents the convexity of the option price in relation to the underlying,

- $\operatorname{Thta}(\Theta)$ : represents the effect of time on the value of the option,

- $\operatorname{Rho}(\rho)$ : represents the effect of the price of the option in relation to the inter- Continuously composed,

- $\operatorname{Vga}(V)$ : represents the effect of the option price on volatility. 
These differents sensitivities are obtained from the results from the theorems (2) and (3). The following corollary gives the sensibilities of the call european option and is the fifth fondamental result of this paper:

Corollary 2 The five sensitivities of the call option for $t \in] 0, T$ [ are defined in the following table:

\begin{tabular}{|l|c|c|}
\hline & & Calls \\
\hline$\Delta$ & $\frac{\partial C}{\partial S}$ & $F\left(d_{1}\right)$ \\
\hline$\Gamma$ & $\frac{\partial^{2} C}{\partial S^{2}}$ & $\frac{F^{\prime}\left(d_{1}\right)}{S \sigma \sqrt{\lambda_{H}(T-t)}}$ \\
\hline$V$ & $\frac{\partial C}{\partial \sigma}$ & $S F^{\prime}\left(d_{1}\right) \sqrt{\lambda_{H}(T-t)}$ \\
\hline$\Theta$ & $\frac{\partial C}{\partial t}$ & $-\frac{S F^{\prime}\left(d_{1}\right) \sigma}{2 \sqrt{\lambda_{H}(T-t)}}-r K e^{-r(T-t)} F\left(d_{2}\right)$ \\
\hline$\rho$ & $\frac{\partial C}{\partial r}$ & $K \sqrt{\lambda_{H}(T-t)} e^{-r(T-t)} F\left(d_{2}\right)$ \\
\hline
\end{tabular}

where $F($.$) is defined in theorem 2$.

Proof. We derive the formula of the call in general, so that $y$ is an arbitrary real variable. So we have

$$
\frac{\partial C}{\partial z}=\frac{\partial S}{\partial z} F\left(d_{1}\right)+S \frac{\partial F\left(d_{1}\right)}{\partial z}-\frac{\partial K e^{-r(T-t)}}{\partial z} F\left(d_{2}\right)-K e^{-r(T-t)} \frac{\partial F\left(d_{2}\right)}{\partial z}
$$

yet

$$
\begin{aligned}
\frac{\partial F\left(d_{2}\right)}{\partial z} & =\frac{1}{\sqrt{2 \pi}} \exp \left(-\frac{d_{2}^{2}}{2}\right) \frac{\partial d_{2}}{\partial z} \\
& =\frac{1}{\sqrt{2 \pi}} \exp \left(-\frac{\left(d_{1}-\sigma \sqrt{\lambda_{H}(T-t)}\right)^{2}}{2}\right) \frac{\partial d_{2}}{\partial z} \\
& =\frac{1}{\sqrt{2 \pi}} \exp \left(-\frac{d_{1}^{2}}{2}\right) \exp \left(d_{1} \sigma \sqrt{\lambda_{H}(T-t)}\right) \exp \left(-\frac{\sigma^{2} \lambda_{H}(T-t)}{2}\right) \frac{\partial d_{2}}{\partial z} \\
& =\frac{1}{\sqrt{2 \pi}} \exp \left(-\frac{d_{1}^{2}}{2}\right) \exp \left(\ln \left(\frac{S}{K}\right)+r(T-t)\right) \frac{\partial d_{2}}{\partial z} \\
& =\frac{1}{\sqrt{2 \pi}} \exp \left(-\frac{d_{1}^{2}}{2}\right) \frac{S}{K} \exp (r(T-t)) \frac{\partial d_{2}}{\partial z}
\end{aligned}
$$

We substitute the latter in (53) and we obtain:

$$
\frac{\partial C}{\partial z}=\frac{\partial S}{\partial z} F\left(d_{1}\right)-\frac{\partial K e^{-r(T-t)}}{\partial z} F\left(d_{2}\right)+S F^{\prime}\left(d_{1}\right) \frac{\left.\partial \sigma \sqrt{\lambda_{H}(T-t)}\right)}{\partial z}
$$

From the same, the following corollary gives the sensibilities of the put european option and is the sixth fondamental result of this paper:

Corollary 3 The five sensitivities of the put option for $t \in] 0, T$ [ are defined in the following table:

\begin{tabular}{|l|c|c|}
\hline & & Puts \\
\hline$\Delta$ & $\frac{\partial P}{\partial S}$ & $F\left(d_{1}\right)-1$ \\
\hline$\Gamma$ & $\frac{\partial^{2} P}{\partial S^{2}}$ & $\frac{F^{\prime}\left(d_{1}\right)}{S \sigma \sqrt{\lambda_{H}(T-t)}}$ \\
\hline$V$ & $\frac{\partial P}{\partial \sigma}$ & $S F^{\prime}\left(d_{1}\right) \sqrt{\lambda_{H}(T-t)}$ \\
\hline$\Theta$ & $\frac{\partial P}{\partial t}$ & $-\frac{S F^{\prime}\left(d_{1}\right) \sigma}{2 \sqrt{\lambda_{H}(T-t)}}+r K e^{-r(T-t)} F\left(-d_{2}\right)$ \\
\hline$\rho$ & $\frac{\partial P}{\partial r}$ & $-K \sqrt{\lambda_{H}(T-t)} e^{-r(T-t)} F\left(-d_{2}\right)$ \\
\hline
\end{tabular}

with $F($.$) defined in theorem 2$.

Proof. See the similary proof of corollary 2. 


\section{Conclusion}

We have in this paper solved the fractional Black-Scholes equation with the hurst index $H \in[0,1]$ our approach consisted to transform the equation of fractional Black-Scholes into a heat equation generalized. For this purpuse, we prove some results giving the resolution of a stochastic differential equation directed by the fractional Brownian motion. We also compare our results with the Ciprian Necula's (Necula, C. (2002)) results. We have noticed that, in the case of the standard fractional brownian motion, the price of the option at the given moment $t \in] 0, T$ [ depends not only on $\lambda_{H}(T-t)$, but also on the stock price of an action $S_{t}$. We have obtained the formula of the call and of the European put, as well as the volatility formula implicit in the option and we have also analysed the sensitivity indicators of the option on financial market.

\section{References}

Ansel, J. P., \& Stricker, C. (1992). Laws of martingale, densities and Decomposition of Föllmer- Schweizer. Ann. Inst. H. PoincarProbab. Statist, 28(3), 375-392.

Arcadius, Y., \& Akossou, Y. J. (2014). Solving the Black Scholes Equation using a Finite Difference implicit Method.

Bender, C., \& Marquardt, T. (2008). Stochastic calculus for convoluted Levy processes. Bernoulli, 14, 499-518. https://doi.org/10.3150/07-BEJ115

Biagini, F., Hu, Y., Øksendal, B., \& Zhang, T. (2008). Stochastic Calculus for Fractional Brownian Motion and Applications. Probability and Its Applications. Springer.

Biagini, F., Yaozhong, H., Øksendal, B., \& Tusheng, Z. (2008a). Stochastic calculus for fractional Brownian motion and applications. Probability and its Applications (New York). Springer-Verlag London Ltd., London. ISBN 978-185233-996-8. https://dx.doi.org/10.1007/978-1-84628-797-8

Black, F., \& Myron, S. (1973). The pricing of options and corporate liabilities. Journal of Political Economy, 81(3), 637-654. https://doi.org/10.1086/260062

Bright, O., Angela, I., \& Chukwunezu. (2014). On the Solution to a Fractional Black-Scholes Equation for the Price of an Option. International Journal of Mathematical Analysis and Applications, 1(3), 38-42.

Cheridito, P. (2003). Arbitrage in fractional Brownian motion models. Finance Stoch., 7(4), 533-553. https://doi.org/10.1007/s007800300101

Coviello, R., Cristina, D. G., \& Russo, F. (2011). On stochastic calculus related to financial assets without semimartingales. Bull. Sci. Math., 135(6-7), 733-774. https://doi.org/10.1016/j.bulsci.2011.06.008

Cox, D. R. (1984). Long-range dependence. A review, in Statistics: an appraisal, H.A. David and H.T. David, eds., Iowa State University Press, 55-74.

Delbaen, F., \& Schachermayer, W. (1994). A general version of the fundamental theorem of asset pricing. Math. Ann., 300(3), 463-520. https://doi.org/10.1007/BF01450498

Gael, D. (2012). Applications du mouvement brownien fractionnaire ?l'molution des engagements sur des contrats en unit?de couple. Mmoire de la chaire d'actuaritat. 111 pages.

Guasoni, P. (2006). No arbitrage under transaction costs, with fractional Brownian motion and beyond. Math. Finance, 16(3), 569-582. https://doi.org/10.1111/j.1467-9965.2006.00283.x

Inkaya, A. (2011). Option pricing fractional Brownian motion. ksendal, B., Biagini, F., \&. al. (2008). Stochastic calculus for fractional Brownian motion and applications. Springer. 94

Kavin, S. (2010). Numerical Methods For Derivative Pricing with Applications to Barrier Option. Thesis presented to the University of Waterloo in fullment of the thesis requirement for the degree of Master of Science in Computational Mathematics Waterloo, Ontario, Canada.

Lindstron, T., Øksendal, B., \& Uboe. (1992). Wick multiplication and Ito-Skorohod stochastic differential equations in S. Cambridge Univ press, pp.183-206.

Mandelbrot, B., \& Van Ness, J. (1968). Fractional Brownian motions, fractional noises and applications. SIAM Review, $10,422-437$.

Malliavin, P., \& Thalmaier, A. (2006). Stochastic calculus of variations in mathematical finance. Springer Finance. Springer-Verlag, Berlin.

Mandelbrot, B. (1971). When can price be arbitraged efficiently? A limit to the validity of the random walk and martingale 
models. The Review of Economics and Statistics, 53(3), 225-236.

Marquardt, T. (2006). Fractional Lvy processes with an application to long memory moving average processes. Bernoulli, 12(6), 1099-1126.

Millet, A. (2000). Stochastic computation 2, Applied mathematics Finance. University of Paris 1.

Necula, C. (2002). Option pricing in a fractional Brownian motion environment. Academy of economic studies bucharest: romania.

Nualart, D. (1995). The Malliavin Calculus and Related Topics. Springer. https://doi.org/10.1007/978-1-4757-2437-0

$\emptyset$ ksendal, B., Biagini, F., \&. al.(2008). Stochastic calculus for fractional Brownian motion and applications, Springer. 94.

Peters, E. E. (1996). Chaos and Order in the Capital Markets: A New View of Cycles, Prices, and Market Volatility. Number vol. 1 in Chaos and Order in the Capital Markets: A New View of Cycles, Prices, and Market Volatility. Wiley.

Robert, J., Elliott, \& John, V. D. H. (2003). A general white noise theory and applications to finance. Math. Finance, 13, 301-330. https://doi.org/10.1111/1467-9965.00018

Rogers, L. C. G. (1997). Arbitrage with fractional Brownian motion.

Russo, F. M., \& Vallois, P. (1993). Forward, backward and symmetric stochastic integration. Probability Theory and Related Fields, 97(3), 403-421. https://doi.org/10.1007/BF01195073

Samorodnitsky, G., \& Taqqu, M. S. (1994). Stable non-Gaussian random processes. Chapman and Hall, New York.

Tikanmä ki, H., \& Mishura, Y. (2011). Fractional Lvy processes as a result of compact interval integral transformation. Stoch. Anal. Appl., 29(6), 1081-1101.

Willinger, W., Taqqu, M. S., Leland, W. E., \& Wilson, D. V. (1995). Self-similarity in high-speed packet traffic: analysis and modeling of Ethernet traffic measurements. Statistical Science, 10, 67-85. https://doi.org/10.1214/ss/1177010131

\section{Copyrights}

Copyright for this article is retained by the author(s), with first publication rights granted to the journal.

This is an open-access article distributed under the terms and conditions of the Creative Commons Attribution license (http://creativecommons.org/licenses/by/4.0/). 